\title{
Increasing the sensitivity of controlled-source electromagnetics with synthetic aperture
}

\author{
Y. Fan ${ }^{1}$, R. Snieder ${ }^{2}$, E. Slob ${ }^{3}$, J. Hunziker ${ }^{3}$, J. Singer ${ }^{4}$, J. Sheiman ${ }^{5}$, and M. Rosenquist ${ }^{5}$
}

\begin{abstract}
Controlled-source electromagnetics (CSEM) has been used as a derisking tool in the hydrocarbon exploration industry. We apply the concept of synthetic aperture to the lowfrequency electromagnetic field in CSEM. Synthetic aperture sources have been used in radar imaging for many years. Using the synthetic aperture concept, big synthetic sources can be constructed by adding the response to small sources (building blocks) in different ways, and consequently, big sources with different radiation patterns can be created. We show that the detectability of hydrocarbons is significantly enhanced by applying synthetic aperture to CSEM data. More challenging targets such as deep reservoirs ( $4 \mathrm{~km}$ below sea floor) can be detected. The synthetic aperture technique also increases the sensitivity of the field to subsurface targets in the towing streamer acquisition. We also show that a pseudovertical source (orthogonally distributed dipole pairs) can be constructed synthetically, and that the detection capability of this pseudovertical source is increased by applying field steering. The synthetic aperture concept opens a new line of research in CSEM, with the freedom to design suitable synthetic aperture sources for a given purpose.
\end{abstract}

\section{INTRODUCTION}

Low-frequency controlled-source electromagnetics (CSEM) has been used in the oil industry as a derisking tool to distinguish hydrocarbon reservoirs since the beginning of this century. In hydrocarbon exploration, CSEM is also referred to as seabed logging
(Ellingsrud et al., 2002). The fundamental assumption of using CSEM as a detector of hydrocarbons is that porous rocks are resistive when saturated with gas or oil, but conductive when filled with water. The history and detailed description of CSEM is given by MacGregor and Sinha (2000), Edwards (2005), and Constable and Srnka (2007).

Although there are many successful case studies showing the ability of CSEM to detect hydrocarbon reservoirs, this method has not been completely accepted by the industry as an exploration tool. The fundamental reason of the limitations in using CSEM is the diffusive nature of the electromagnetic field in conductive media such as sea water and the subsurface below it. Because of diffusion, the electromagnetic field decays rapidly in space, and consequently, the secondary field refracted from the target usually is much smaller than the background field (the received field without the target). Because only the secondary field from the target carries information about the target, the interpretation of the target signature is problematic if the target field is hidden in the background field. Therefore, most of the successful applications of CSEM are in deep water $(>1 \mathrm{~km})$, with a shallow target $(<2.5 \mathrm{~km})$, with a large horizontal extent (several kilometers), and few other resistors in the background. Even with these criteria, the anomaly in the recorded field due to the hydrocarbon reservoir is small.

Fan et al. (2010) showed that the detectability of hydrocarbon reservoirs increases dramatically by forming a synthetic aperture source for CSEM. In this paper, we explore this concept in more detail and show some of the valuable applications and improvements to CSEM exploration. Synthetic aperture is a concept that has been widely used in the radar and sonar community e.g., (Cutrona, 1975; Barber, 1985; Riyait et al., 1995; Bellettini and Pinto, 2002; Ralston et al., 2007; Zhou et al., 2009). The basic idea of the synthetic aperture concept is to use the interference of the fields from different

Manuscript received by the Editor 23 May 2011; revised manuscript received 18 August 2011; published online 23 February 2012.

${ }^{1}$ Colorado School of Mines, Center for Wave Phenomena, Golden, Colorado, USA; Shell International Exploration \& Production, Houston, Texas, USA E-mail: uanzhong.fan@ shell.com.

${ }^{2}$ Colorado School of Mines, Center for Wave Phenomena, Golden, Colorado, USA. E-mail: rsnieder@mines.edu.

${ }^{3}$ Delft University of Technology, Department of Geotechnology, Delft, The Netherlands. E-mail: e.c.slob@tudelft.nl; j.w.hunziker@tudelft.nl.

${ }^{4}$ Shell International Exploration \& Production, Houston, Texas, USA; presently at Fugro General Geophysical Services, Leidschendam, The Netherlands. E-mail: Johannes.singer@gmx.net.

${ }^{5}$ Shell International Exploration \& Production, Houston, Texas, USA. E-mail: johnathan.sheiman@shell.com; mark.rosenquist@shell.com.

(c) 2012 Society of Exploration Geophysicists. All rights reserved. 
sources to construct a big synthetic source (aperture) which has a special radiation pattern designed for a specific purpose.

We illustrate the concept with a simple example. In a lossless homogeneous space, the frequency-domain 3D Green's function is $G(r, \omega)=e^{i k r} / r$ for wave propagation. The real part of the Green's function from a point source located at the origin is shown in the upper panel of Figure 1. In this example, we use a frequency of $0.25 \mathrm{~Hz}$, a medium velocity of $866 \mathrm{~m} / \mathrm{s}$. We next construct an $10 \mathrm{~km}$ long elongated source by adding 200 uniformly distributed point sources from $x=-5 \mathrm{~km}$ to $5 \mathrm{~km}$, all at $z=0 \mathrm{~km}$. Apart from contributions from the edges of the source array, the elongated source emits a plane wave that propagates in the vertical direction (lower panel of Figure 1). Compared to a physical source $10 \mathrm{~km}$ long, one has more freedom in building a big source synthetically by adding the fields emitted by small sources. For example, a linear phase shift along the source array can be applied to the individual sources before the summation, and as a result the total field is steered in a certain angle. This is illustrated by the upper panel of Figure 2. Similarly, the total field can be focused in a location if appropriate phase shifts are applied to the individual sources, as shown in the lower panel of Figure 2. Steering and focusing using synthetic aperture has been used for wave problems such as radar and ultrasound imaging (Berson et al., 1981; Lu et al., 1994; Aguttes et al., 2000; Wang et al., 2009; Korobov et al., 2010).

Fan et al. (2011) have shown that diffusive fields can also be steered and focused by applying an appropriate phase shift and amplitude weighting. Consequently, one can extend the synthetic aperture concept to CSEM, where the electromagnetic fields propagate diffusively. The basic idea is that a diffusive field can be viewed as a highly attenuating wave with dispersion in phase velocity and attenuation. In fact, the similarities in the mathematical expressions of wave propagation and diffusion can be found in the literature (Kunetz, 1972; Isaev and Filatov, 1981; Filatov, 1984; Lee et al., 1989; O'Leary et al., 1992; Boas et al., 1993, 1994). Also, the interference of diffusive fields has been widely used in physics
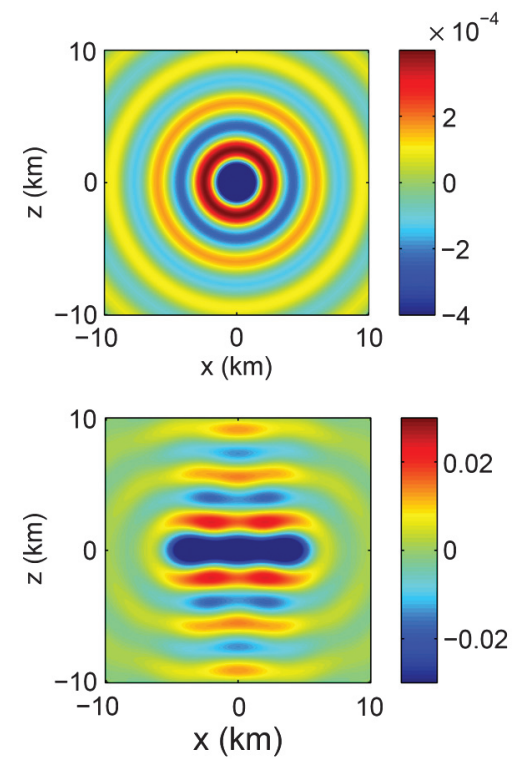

Figure 1. Upper panel: real part of the Green's function $G(r, \omega)=$ $e^{i k r} / r$ for a point source of waves; Lower panel: an elongated source, $10 \mathrm{~km}$ long, created by adding $\operatorname{Re}(G)$ from 200 point sources between $x=-10 \mathrm{~km}$ and $x=10 \mathrm{~km}$.
(Schmitt et al., 1992, 1993; Knuttel et al., 1993; Yodh and Chance, 1995; Wang and Mandelis, 1999).

In this paper, we show that the imprint of the hydrocarbon reservoir on measured fields can be dramatically increased by applying field steering to CSEM data. This enhances the ability to detect more challenging reservoirs. We show that a deep target $(4 \mathrm{~km}$ below the sea floor) can be detected with field steering. The synthetic aperture technique also increases the sensitivity of the measured field to subsurface targets when receivers are located in streamers towed near the sea surface. We also show that the pseudovertical source (orthogonally distributed dipole pairs) can be constructed synthetically. The detection capability with a pseudovertical source is increased by applying field steering. In practice, one can design a variety of synthetic sources depending on the goal of the survey. As is common for CSEM, the data and analysis in the following examples are treated in the frequency domain.

\section{FIELD STEERING}

A general formula for constructing a synthetic aperture source $S_{A}$ is

$$
S_{A}(\mathbf{r}, \omega)=\sum_{n=1}^{N} a_{n} e^{i \phi_{n}} s\left(\mathbf{r}, \mathbf{r}_{n}, \omega\right)
$$

At a single angular frequency $\omega$, a synthetic source at location $\mathbf{r}$ is formed by superposition of the spatially distributed sources that are located from $\mathbf{r}_{1}$ to $\mathbf{r}_{N}$ with an amplitude weighting $a_{n}$ and a phase shift $\phi_{n}$. In expression $1, s\left(\mathbf{r}, \mathbf{r}_{n}, \omega\right)$ is the individual source function. Consistent with typical CSEM surveys, the sources are assumed to be continuously distributed along a line (taken to be the $x$ axis). We apply a linear phase shift to the individual source in the line to steer the field to an designed direction. The total field can be represented as
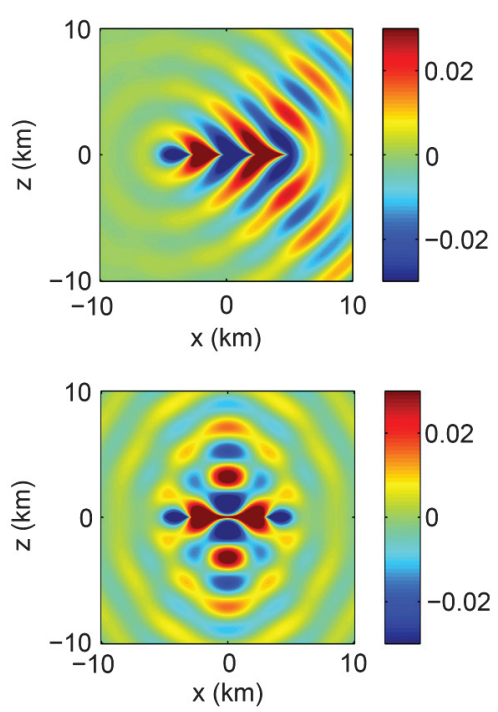

Figure 2. Upper panel: an elongated source, $10 \mathrm{~km}$ long, created by adding $\operatorname{Re}(G)$ from 200 point sources with field steering at $45^{\circ}$; Lower panel: an elongated source, $10 \mathrm{~km}$ long, created by adding $\operatorname{Re}(G)$ from 200 point sources with field focusing at $x=0 \mathrm{~km}$ and $z=-3 \mathrm{~km}$. 


$$
S_{A}(x, \omega)=\sum_{n=1}^{N} e^{-i c_{1} \alpha \Delta x_{n}} e^{-c_{2} \alpha \Delta x_{n}} s\left(x, x_{n}, \omega\right),
$$

where $\alpha=\sqrt{\omega \mu \sigma / 2}$ is the real or imaginary part of the wave number $k$ for the diffusive electromagnetic field, $\mu$ is the permeability, $\sigma$ is the conductivity, $c_{1}$ is a coefficient to control the steering angle (phase change), $c_{2}$ is a coefficient to compensate the energy loss due to the diffusion (amplitude weighting), and $\Delta x_{n}=\left|x_{n}-x_{1}\right|$ is the distance from the source $x_{n}$ to the left edge of the array.

\section{Shallow target}

We first show field steering to a shallow target $(1 \mathrm{~km}$ below the sea floor) to illustrate the dramatic increase of the reservoir anomaly in measured fields. The model we use in this numerical example is shown in Figure 3. A hydrocarbon reservoir with resistivity of $100 \Omega \mathrm{m}$ is centered at the origin, with horizontal extent of $4 \mathrm{~km}$ in the $x$ - and $y$-directions and a thickness of $100 \mathrm{~m}$. The sea water is $1 \mathrm{~km}$ deep with a resistivity of $0.3 \Omega \mathrm{m}$. The subsurface background is a half space with a resistivity of $1 \Omega \mathrm{m}$. The receivers are located at the sea floor and a $100 \mathrm{~m}$ dipole source with a current of $100 \mathrm{~A}$ is continuously towed $100 \mathrm{~m}$ above the receivers. The source current oscillates with a frequency of $0.25 \mathrm{~Hz}$.

Figure 4a shows the inline electrical fields in the presence of the reservoir (dashed line) and without the reservoir (solid line) from a single $100 \mathrm{~m}$ dipole source whose center is located at $x=-6.5 \mathrm{~km}$. There is a slight increase in the field around $x=0 \mathrm{~km}$ when the reservoir is present. This $20 \%$ difference is shown by the ratio of the field with the reservoir to the field without the reservoir (thin solid curve in Figure 4e). We next construct a $5 \mathrm{~km}$ long dipole by superposing the 50 employed sequential sources. This is equivalent to setting $c_{1}=0$ (zero angle steering), $c_{2}=0$ (same weighting), and $N=50$ in equation 2 . The superposition of the fields is exactly the same as the field from a $5 \mathrm{~km}$ long physical dipole source with a current of $100 \mathrm{~A}$. The total $E_{x}$ fields are given by Figure $4 \mathrm{~b}$. The ratio of the fields with and without the reservoir is shown by the dashed curve in panel (e). Although the overall signal strength increases compared to the single $100 \mathrm{~m}$ source (panel (a)), the

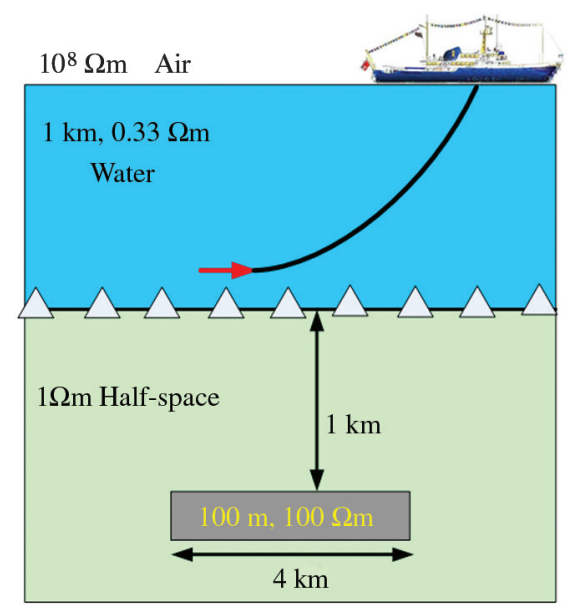

Figure 3. Model used in section "Shallow target." The arrow towed by the boat is the dipole source and the triangles on the sea floor are the receivers. difference in the measured $E_{x}$ fields between the models with and without the reservoir does not significantly increase by using a longer dipole.

We next apply a linear phase shift to the 50 sequential sources using $c_{1}=0.7$ to steer the field to the angle of approximately $45^{\circ}$. The coefficient $c_{1}$ controls the slope of the linear phase shift and can be related to the steering angle $\theta$ (steering direction to the vertical) by $c_{1}=\sin \theta$ (valid for $c_{1}<1$ ) (Fan et al., 2011). Figure $4 \mathrm{c}$ shows the $E_{x}$ field excited by the synthetic aperture source whose field is steered to the right. The ratio of the steered fields is illustrated by the thick solid curve in the bottom panel. This example shows that the detectability of the reservoir significantly increases by steering the field toward the target.

To explain the effectiveness of field steering, we consider how the field steering affects the way in which the electromagnetic field propagates. Figure 5 shows the depth view of the orientation of

a)

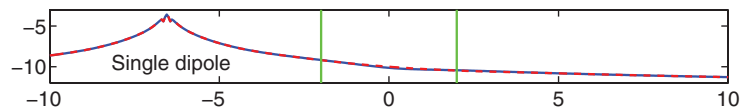

b)

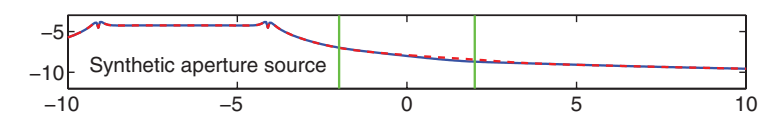

c)

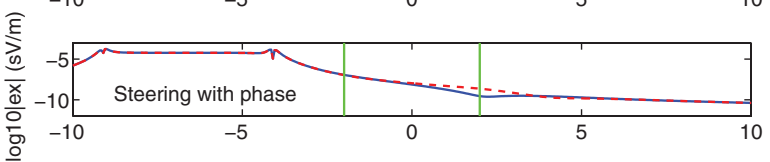

d)
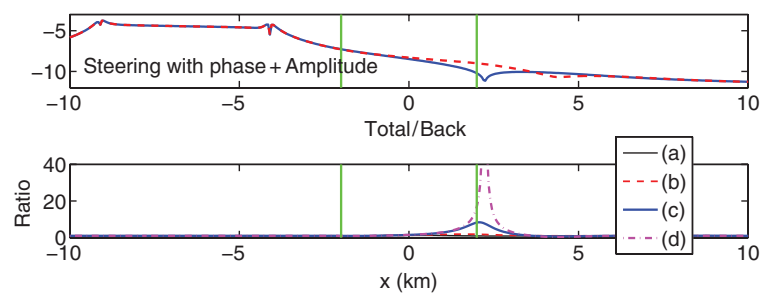

Figure 4. Panels (a-d) show the inline electrical fields with the reservoir (dashed lines) and without the reservoir (solid) for four different sources; a 100 m dipole source (a); a $5 \mathrm{~km}$ dipole source (b); a $5 \mathrm{~km}$ synthetic source obtained from field steering toward the target by the phase shift (c); a $5 \mathrm{~km}$ synthetic source obtained from field steering toward the target by the phase shift and the amplitude compensation. Panel (e) shows the ratio between the fields with and without the reservoir. The four curves in (e) represent the ratios from each of the panels above.
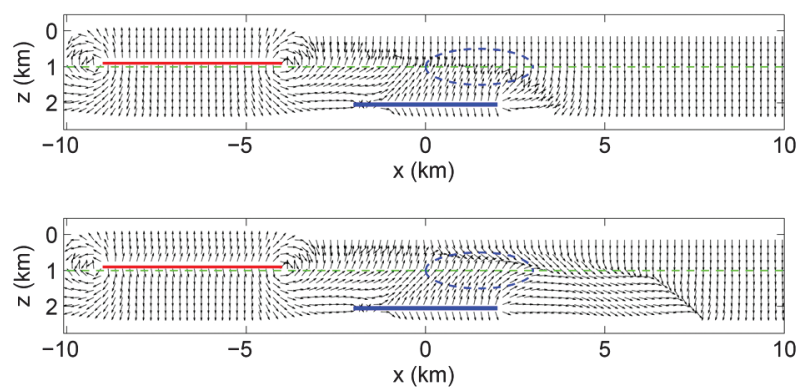

Figure 5. The direction of Poynting vector in the vertical plane through the acquisition line before (upper panel) and after steering (bottom panel). Because the panels show the normalized Poynting vector, all vectors have the same length. 
the Poynting vector before (upper panel) and after (bottom panel) steering. The Poynting vector accounts for the energy flow. The horizontal dashed line at depth $1 \mathrm{~km}$ represents the sea floor. The horizontal bar above the sea floor is the dimension of the synthetic dipole source. The horizontal bar $1 \mathrm{~km}$ below the sea floor shows the extent of the reservoir. The offset range, where the anomaly is present in data, is highlighted by the dashed ellipse. The orientation of the Poynting vector suggests that energy propagates along several paths. In the case without steering (upper panel), right above and below the synthetic aperture source ( $x$ from $-9 \mathrm{~km}$ to $-4 \mathrm{~km})$, the energy propagates vertically away from the source. For small offsets ( $x$ from $-4 \mathrm{~km}$ to $0 \mathrm{~km}$ ), the energy dives in the earth and comes back to the sea floor. There is also energy turned back from the sea water to the sea floor. At the depth close to the sea surface, the net energy flow is dominated by the air wave, which propagates vertically downward. For intermediate offsets ( $x$ between $0 \mathrm{~km}$ and $3 \mathrm{~km}$ ), the upgoing secondary field from the subsurface target is visible. This secondary field, however, interferes with the downgoing airwave and upgoing diving field at the measurement locations (sea floor). For large offsets $(x>3 \mathrm{~km})$, the energy propages vertically downward, which is due to the dominance of the airwave. Overall, there is a small window, around $x=2 \mathrm{~km}$, in which the secondary field shows an anomaly at the measurement level (see Figure 4b).

The lower panel of Figure 5 shows that the steering changes the energy propagation pattern in space. At the measurement level, there is noticeable increase of the upgoing energy. Around $x=2 \mathrm{~km}$, the secondary field from the reservoir propagates upward to the sea floor and has less interference with other arrivals. Below we explain this change in the energy flow.

When the field is steered toward the target, there are two changes in the radiation pattern that lead to the increased imprint of the reservoir in the measured field. First, the portion of the energy

a)

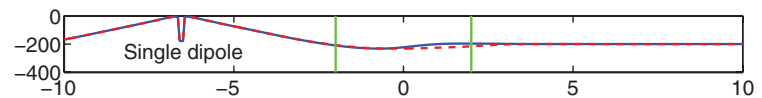

b)

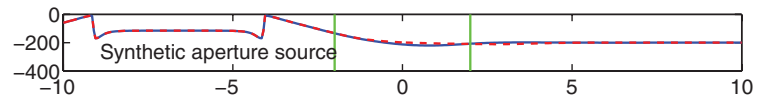

c)

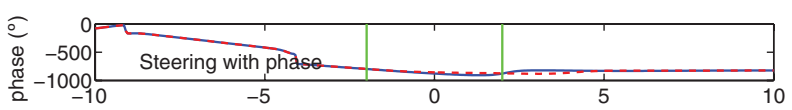

d)

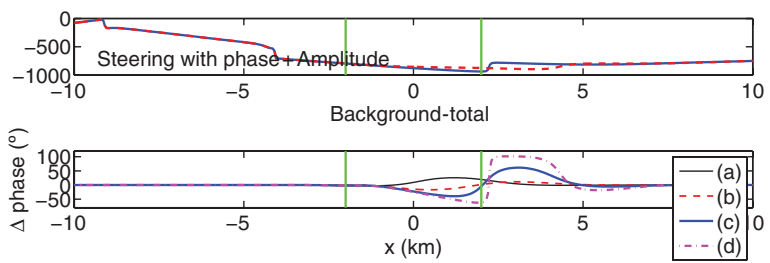

Figure 6. Panels (a-d) show the phase of the inline electrical fields with the reservoir (dashed lines) and without the reservoir (solid) for four different sources; a $100 \mathrm{~m}$ dipole source (a); a $5 \mathrm{~km}$ dipole source (b); a $5 \mathrm{~km}$ synthetic source obtained from field steering toward the target by the phase shift (c); a $5 \mathrm{~km}$ synthetic source obtained from field steering toward the target by the phase shift and the amplitude compensation. Panel (e) shows the phase difference between the fields with and without the reservoir. The four curves in (e) represent the phase difference from each of the panels above. toward the reservoir increases when the field is steered at an angle. Therefore, the secondary field refracted from the reservoir increases. In our synthetic example, this secondary field increases about $30 \%$ (not shown in Figure 4). Second, when the energy is steered away from the vertical direction, the field reflected by the sea surface increases while the airwave decreases. Because the reflected field has a longer propagation path in the sea water than the air wave, it attenuates more, and the total received field decreases due to the reduction of the airwave. Evidence to support the reasoning of the reducing airewaves is that the tail part of the background $E_{x}$ field, which consists mostly of the airwave, decreases on both sides of the source regardless of whether the field is steered to the right or left. Given that the secondary field from the target increases because of the improved illumination, and the background field decreases mainly because of the airwave reduction, the imprint of the reservoir is more pronounced after field steering.

Figure 6 shows the phase of the synthetic aperture. The panels are presented in the same order as Figure 4. The steering of the field significantly increases the phase difference between the model with and without the reservoir as shown in Figure 6e. The explanation of the improved detectability of the reservoir relies on the interference of different arrivals. Because this reasoning is applicable to the phase and amplitude, we only present the magnitude of the field in the following examples.

So far, we have only applied phase shifts to the individual sources to steer the total field to one side of the synthetic source. The attenuation of a diffusive field causes the sources on the opposite side to give a smaller contribution to the synthetic aperture construction because they propagate a greater distance. Because the diffusive field decays exponentially, an exponential weighting term $e^{-c_{2} \alpha \Delta x_{n}}$ in equation 2 is included to compensate for this energy loss, where $c_{2}$ is a constant that controls how much weight each individual sources have. Fan et al. (2011) show that for an homogeneous medium, the best steering is achieved for $c_{2}=c_{1}$, but in the layered model used here we find empirically that the anomaly due to the reservoir is largest when $c_{2}=0.1$. Figure $4 \mathrm{~d}$ shows the fields after including the energy compensation term $e^{-0.1 \alpha \Delta x_{n}}$. This difference is quantified by the ratio of the fields with and without the target and is illustrated by the dashed-dotted line in panel (e).

Why is the optimal value of $c_{2}$ different for a homogeneous medium than for the layered model we use here? As explained earlier, there are two reasons for steering to cause the reservoir imprint to be more pronounced: the increase of the secondary field, and the decrease of the background field. The increase of the secondary field is optimal when $c_{1}=c_{2}$, but the decrease of the background field is more complicated for a variety of reasons. The first reason, as state above, is the reduction of the airwave due to the change of the incident angle. The second reason is the interference between different arrivals, which makes the resulting interference of these arrivals depend in a complicated way on the radiation pattern of the synthetic source and the earth model. There are many different paths for the energy travel from the source to the receivers, as Figure 5 illustrates. Energy can propagate directly from the source to the receiver, be carried by the airwave, be reflected and refracted from the sea floor, or be refracted back from the shallow depth (like a diving wave). There is constructive and destructive interference between these arrivals. When the field is steered, destructive interference can occur in the background field (the dip around $x=2 \mathrm{~km}$ in Figure $4 \mathrm{c}$ and $4 \mathrm{~d}$ ). This dip in the background field creates a 
window through which the secondary target field can be better detected. As the amplitude of each arrivals varies, the amplitude weighing (by the parameter $c_{2}$ ) of individual sources is critical because destructive interference only occurs when the interfering fields are of a comparable strength.

Further research is needed to identify the individual arrivals, to better understand the mechanism of the destructive interference, and optimally design the amplitude weighting for each source. For example, for a more complicated earth model, what is the best combination of $c_{1}$ and $c_{2}$ ? Is the exponential weighting function best at creating the interference window? In practice, however, the earth model is unknown. Therefore, it is most practical to numerically search the optimal parameters of $c_{1}$ and $c_{2}$. Figure 7 is an example of the parameter search for the optimal steering parameters for the model of Figure 4. The figure shows the peak of the anomaly ratio as a function of $c_{1}$ and $c_{2}$. A large number of $c_{1}$ and $c_{2}$ pairs are used in the creation of the synthetic aperture source to find the optimal parameters that give the largest anomaly. This is equivalent to sweeping the field from zero to $90^{\circ}$ while changing the weighting of the individual sources. Figure 7 thus shows the sensitivity of the detected anomaly as a function of $c_{1}$ and $c_{2}$. Because the CSEM data set is relatively small, the computational cost of this parameter search is low. Note that one does not need to acquire new field data to carry out this parameter search. It only requires processing of existing data.

The above example shows that the synthetic aperture technique dramatically increases the difference in electrical field response between the models with and without the reservoir (anomaly of a factor of 40). Strictly speaking, it is not fair to directly compare Figure 1a with the elongated dipole (b-d) because this particular dipole position is not optimal for detection of the reservoir using a small source. For this model, the maximum anomaly from a single dipole is four (instead of 0.2 in panel [a]) if it is located at the optimal position. Even though, the increase of the anomaly by the field steering is significant. Note that this gain is achieved without altering the data acquisition. If noise is added in the above example, the main observation still holds as the coherent fields from the source are still steered. The $\mathrm{S} / \mathrm{N}$ is enhanced because the coherent field increases faster than the noise does when a large synthetic source is constructed. However, the anomaly ratio may not be as big as the example's factor of 40 because the noise is not coherent and cannot be steered.

\section{Deep target}

We have shown that the imprint of the shallow target $(1 \mathrm{~km}$ below the sea floor) dramatically increases after applying field steering. It is logical to think that field steering could help to detect deeper targets. The current deepest reservoir that CSEM can detect is about $3 \mathrm{~km}$. This limitation in depth penetration of CSEM makes this technique not suitable for deep reservoirs. In the following example, we show that by applying field steering to the current CSEM data, a deeper reservoir ( $4 \mathrm{~km}$ below the sea floor) can be clearly detected.

In the following numerical example, a hydrocarbon reservoir with a resistivity of $100 \Omega \mathrm{m}$ is centered at the origin with horizontal extent of $8 \mathrm{~km}$ in the $x$ and $y$ directions and a thickness of $100 \mathrm{~m}$. The reservoir is $4 \mathrm{~km}$ below the sea floor. The source and receiver parameters are the same as used in the previous example. For a better penetration, we choose a lower source frequency of $0.01 \mathrm{~Hz}$. The measured $E_{x}$ fields (single dipole) are shown in Figure 8a. No difference can be seen between the field with the reservoir (solid line) and the field without the reservoir (dashed line). The ratio of these two fields is shown as the solid line in the bottom panel. The vertical lines in each panel represent the edges of the reservoir in the horizontal direction. In fact, no anomaly is visible no matter where the source is located. Figure $8 \mathrm{~b}$ shows the field from a $5 \mathrm{~km}$ long synthetic source with the field steered toward the right. A clear anomaly is present both in the measured field (Figure $8 \mathrm{~b}$ ) and the ratio (Figure $8 \mathrm{c}$ ). In this case, we tested multiple pairs of $c_{1}$ and $c_{2}$, and found the optimal combination to be $c_{1}=3.4$ and $c_{2}=0.1$.

As described above, $c_{1}$ can be related to the incident angle by $c_{1}=\sin \theta$ when $c_{1}<1$. But what happens if $c_{1}$ becomes larger

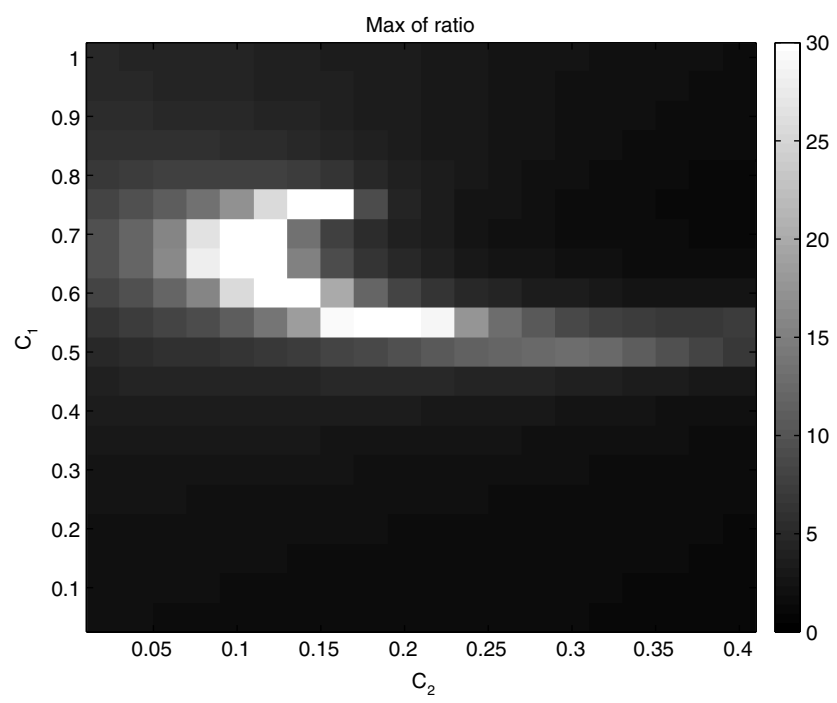

Figure 7. The peak of the anomaly ratio as a function of the steering parameters $c_{1}$ and $c_{2}$.

a)

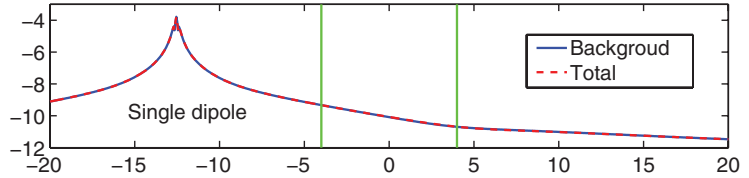

b)
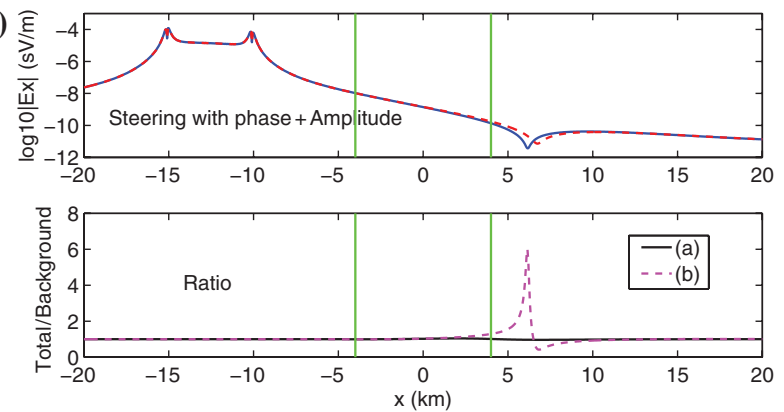

Figure 8. Panels (a) and (b) show the inline electrical field with the reservoir (red dashed lines) and without the reservoir (black solid lines) for two different sources; a single dipole source (a) and a $5 \mathrm{~km}$ synthetic source obtained from field steering toward the reservoir by the phase shift and the amplitude compensation (b). Bottom panel shows the ratio between the fields with and without the reservoir. The two curves in the bottom panel represent the ratios from each of the panels above. 
than one? We first answer this question for wave propagation. For mathematical simplicity, we analyze a $2 \mathrm{D}$ wavefield in a homogeneous medium with $k_{x}^{2}+k_{z}^{2}=\omega^{2} / v^{2}$, where $v$ is the wave velocity. With field steering, the horizontal wavenumber becomes $k_{x}=c_{1} \omega / v$, making the vertical wavenumber $k_{z}= \pm \omega \sqrt{1-c_{1}^{2}} / v$. When $c_{1}$ is less than one, it is related to the steering angle by $c_{1}=$ $\sin \theta$ and $k_{z}=\omega \cos \theta / v$. When $c_{1}>1$, the vertical wave number $k_{z}$ is imaginary and the total field is evanescent. For a diffusive field in a homogeneous medium, $k_{x}^{2}+k_{z}^{2}=i \omega / D$, with $D$ the diffusion constant. With steering, the horizontal wave number is $k_{x}=$ $c_{1} \sqrt{i \omega / D}$, while the vertical wavenumber is $k_{z}=$ $\pm \sqrt{i \omega / D-c_{1}^{2} i \omega / D}= \pm \sqrt{i \omega / D\left(1-c_{1}^{2}\right)}$. When $c_{1}>1, k_{z}$ is still complex, but the decay rate in the $z$ direction depends on the value of $c_{1}$. When $c_{1}>1$, it thus controls the amplitude weighting. As we state above, the amplitudes for different arrivals are crucial to create the destructive interference in the field. In this case, $c_{1}$ and $c_{2}$ control the amplitude weighting and we find empirically that the combination of $c_{1}=3.4$ and $c_{2}=0.1$ creates this destructive interference window that makes it possible to detect a reservoir at $4 \mathrm{~km}$ depth.

\section{Shallow sources and ocean bottom receivers}

In current CSEM surveys, the source usually is towed close to the sea floor to minimize the dissipative loss in sea water. Towing a source at depth can be technically challenging and expensive. Because field steering can increase the imprint of the reservoir dramatically, we show the feasibility of towing the source close to the sea surface while the receivers are still located at the sea floor. Note that if the water depth is too big, near-surface towing obviously can not work because the energy dissipates in the water. But in relatively shallow water, near-surface towing can be applicable considering the increased detectability by applying field steering. In the following example, we use a water depth of $500 \mathrm{~m}$, but this is not a limiting case. Further research is needed to investigate the maximum water depth for a given target for near-surface towing. A modeling feasibility study can be carried out to test if near-surface towing is appropriate for the given water depth and given target (i.e., size, depth and conductivity) following the method we use below.

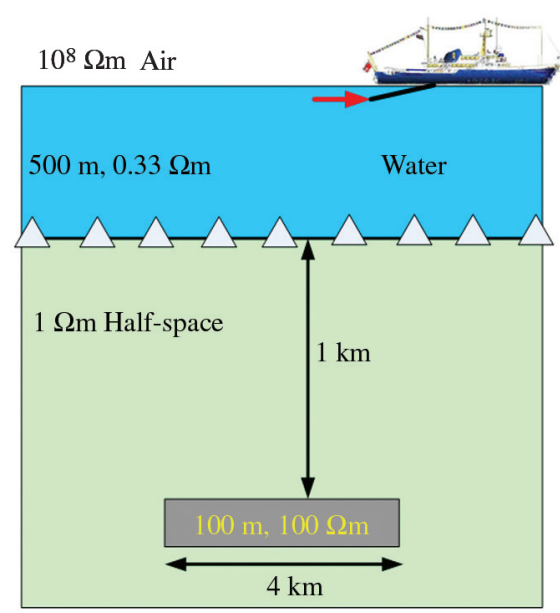

Figure 9. The model used in section "Shallow sources and ocean bottom receivers." The source is towed either $10 \mathrm{~m}$ or $0 \mathrm{~m}$ below the sea surface.
The model used in this example is shown in Figure 9. A hydrocarbon reservoir with the resistivity of $100 \Omega \mathrm{m}$ is centered at the origin at a depth of $1 \mathrm{~km}$ below the sea floor, a horizontal extent of $4 \mathrm{~km}$ in the $x$ - and $y$-directions, and a thickness of $100 \mathrm{~m}$. The source is a $100 \mathrm{~m}$ dipole source with 100 A current, towed $10 \mathrm{~m}$ below the sea surface, oscillating at a frequency of $0.25 \mathrm{~Hz}$. The receivers are located on the sea floor. In Figure 10a, the measured $E_{x}$ fields from the single dipole show no difference between the field with the reservoir (solid line) and the field without the reservoir (dashed line) because the airwave dominates the measured fields. The ratio of the background and the total field is shown by the solid line in Figure 10d. Figure 10b shows the background and total fields from a $5 \mathrm{~km}$ long synthetic source, whose field is steered downward (zero steering). The overall field strength increases, but no imprint of the reservoir can be seen. The ratio of the fields is shown by the dashed line in the bottom panel. Next, we steered the field toward the right using $c_{1}=0.7$ and $c_{2}=0.1$. A clear anomaly is then seen in Figure 10c, with the ratio of the background field and the total field from panel $\mathrm{c}$ indicated by the dashed-dotted line in the bottom panel. Note that, in this case, the field with the reservoir is smaller than the field without reservoir. The definition of the ratio in the bottom panel is different from those used in the previous examples. Instead of the ratio of the total field and the background field, we show the inverse ratio as the total field is smaller than the background field. The fact that the total field is smaller than the background field is an indication of the destructive interference between the target field (secondary field) and the airwave. The phase anomaly (not shown) of the synthetic aperture is also opposite from the one shown in Figure 6e. This is also an indication of a different interference from the one in Figure 6.

We have shown the case where the source is towed $10 \mathrm{~m}$ below the sea surface. To take this one step further, we also test the

a)

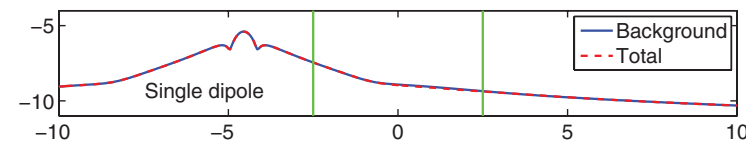

b)

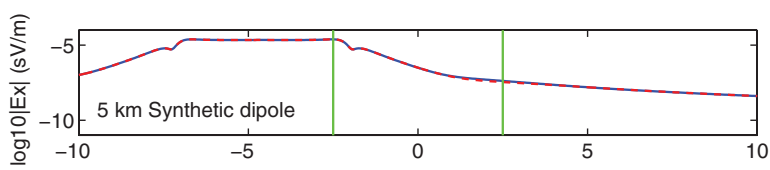

c)

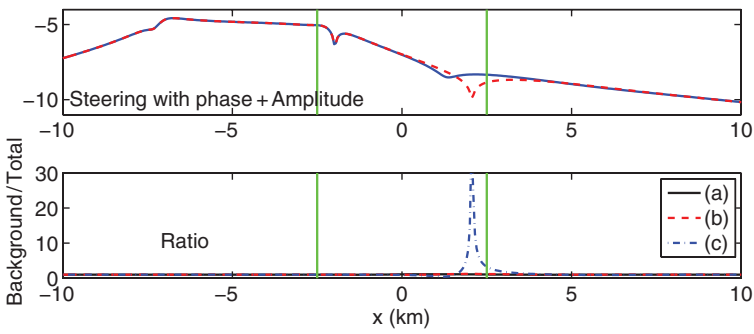

Figure 10. Panels (a-c) show the inline electrical field with the reservoir (dashed lines) and without the reservoir (solid lines) for three different sources; a single dipole source (a); a $5 \mathrm{~km}$ synthetic source (b); and a $5 \mathrm{~km}$ synthetic source obtained from field steering toward the reservoir by the phase shift and the amplitude compensation (c). Panel (d) shows the ratio between the fields with and without the reservoir. The three curves in (d) represent the ratios from each of the panels above. In this system, the dipole source is towed $10 \mathrm{~m}$ below the sea surface and receivers are at the sea floor. 
extreme case of towing the source at the sea surface. As shown in Figure 11, towing the source at the sea surface does not result in a significant difference compared with towing the source at $10 \mathrm{~m}$ in depth. The above two examples show that by applying field steering, CSEM acquisition can be simplified by towing the source close to, or at, the sea surface.

\section{Shallow sources and receivers}

While in the standard CSEM acquisition the receivers are located at the sea floor, recent research shows that the streamer acquisition can also be applied to the CSEM survey (Edwards, 2005; Anderson and Mattson, 2010; McKay et al., 2011). The streamer system has the potential of reducing acquisition cost and providing denser receiver spacing. The dense receiver spacing is crucial for many techniques such as CSEM migration (Zhdanov et al., 1995, 1996; Zhdanov and Traynin, 1997), up-down decomposition, and CSEM interferometry (Wapenaar et al., 2008; Fan et al., 2009; Hunziker et al., 2009, 2010). One drawback, however, is the bigger loss of energy in the sea water because the source and receiver are near the sea surface. Consequently, the sensitivity to the subsurface target is reduced. In this section, we show that by constructing appropriate synthetic aperture, the sensitivity to the subsurface target can be significantly increased in the streamer system.

The near-surface source-and-receiver towing system is shown in Figure 12. The model and source parameters are the same as those used in the previous section. The only difference is that the receivers are towed together with the source at the same depth, instead of being placed on the sea floor.

As in the previous section, we showed two examples: towing the source and receivers $10 \mathrm{~m}$ below the sea surface (Figure 13) and towing them on the sea surface (Figure 14). Neither the single source fields (Figures 13a and 14a) nor the zero steering fields

a)

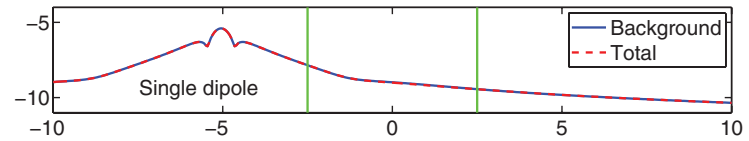

b)

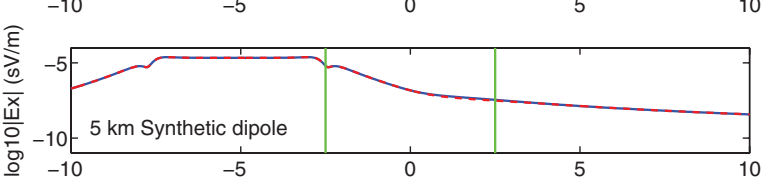

c)
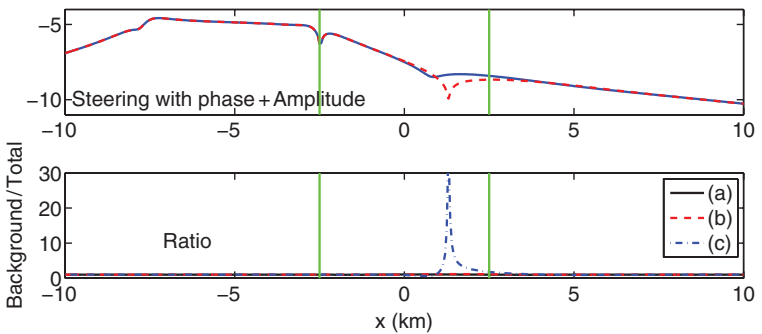

Figure 11. Panels (a-c) show the inline electrical field with the reservoir (dashed lines) and without the reservoir (solid lines) for three different sources; a single dipole source (a); a $5 \mathrm{~km}$ synthetic source (b); a $5 \mathrm{~km}$ synthetic source obtained from field steering toward the reservoir by the phase shift and the amplitude compensation (c). Panel (d) shows the ratio between the fields with and without the reservoir. The three curves in (d) represent the ratios from each of the panels above. In this system, the dipole source is towed at the sea surface and receivers are at the sea floor.
(Figures 13b and 14b) show a clear imprint of the reservoir. For the steered field shown in Figures $13 \mathrm{c}$ and $14 \mathrm{c}$, constructed with $c_{1}=0.7$ and $c_{2}=0.1$, the imprint of the reservoir is clearly visible. The corresponding ratios of the background fields and total fields are shown in Figures 13d and 14d.

These two examples show that the drawback of a near-surface streamer system can be reduced by applying field steering. As described in the previous section, this near-surface towing acquisition can only be applicable when the water is not too deep. While the examples shown here give a proof of concept, further research is needed to investigate the feasibility of using near-surface

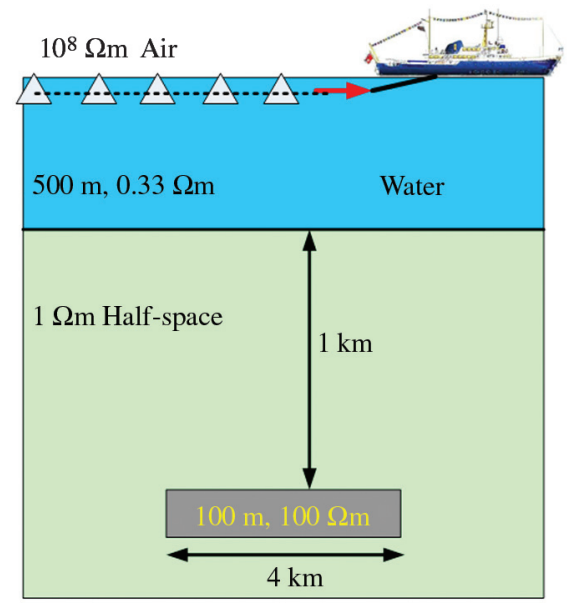

Figure 12. The model used in the section "Shallow towing system for the source and receivers." The source and receivers are towed either $10 \mathrm{~m}$ or $0 \mathrm{~m}$ below the sea surface.

a)

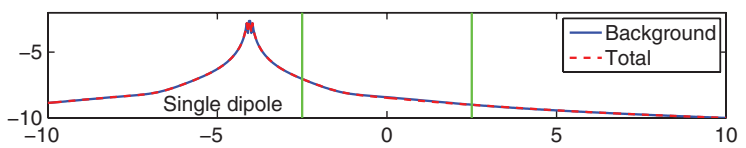

b) $\widehat{\varepsilon}$

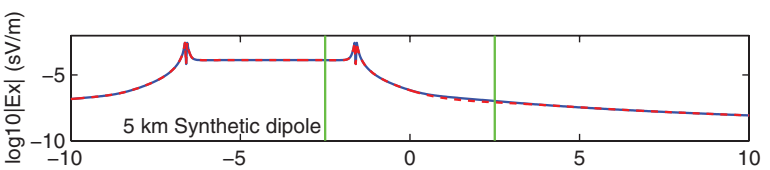

c)
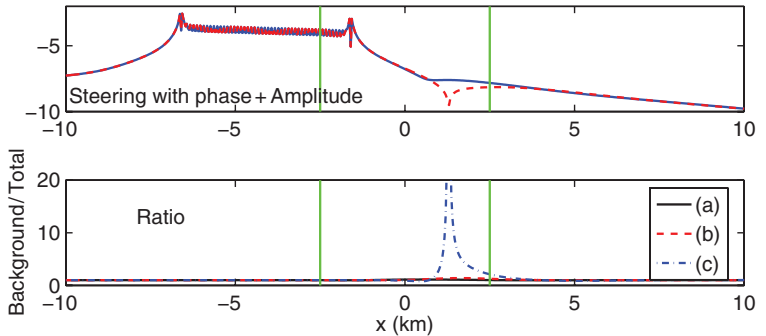

Figure 13. Panels (a-c) show the inline electrical field with the reservoir (dashed lines) and without the reservoir (solid lines) for three different sources; a single dipole source (a); a $5 \mathrm{~km}$ synthetic source (b); a $5 \mathrm{~km}$ synthetic source obtained from field steering toward the reservoir by the phase shift and the amplitude compensation (c). Panel (d) shows the ratio between the fields with and without the reservoir. The three curves in (d) represent the ratios from each of the panels above. In this system, both the dipole source and receivers are $10 \mathrm{~m}$ below the sea surface. 
source-and-receiver towing system for a given model (i.e., the relationship between the water depth and target information, the near-surface background noise and streaming noise).

\section{Real data example}

So far, we have applied field steering to simple synthetic models. Next, we apply field steering to real data. In real data, the field without the reservoir is defined as the measured field at a reference site under which there is no reservoir. Figure 15 a shows the inline electrical fields at $0.25 \mathrm{~Hz}$ for the locations with (dashed) and without (solid) the reservoir. There is a slight difference in the measured fields between $x=6 \mathrm{~km}$ and $x=10 \mathrm{~km}$. The reservoir is known to be located between $x=3 \mathrm{~km}$ and $x=6 \mathrm{~km}$ (as indicated by the vertical lines in Figure 15) and $2.5 \mathrm{~km}$ below the sea floor. The sea water is about $2 \mathrm{~km}$ deep. The reservoir is laterally about $1 \mathrm{~km}$ away from the towline. The corresponding ratio of the two fields is shown by the solid curve in panel (d). The anomaly in the field near the reservoir is approximately $20 \%$ around the offset $x=8 \mathrm{~km}$. Beyond offsets of $10 \mathrm{~km}$, the ratio oscillates as the field reaches the noise level and is no longer reliable. At the small offset range, the wild oscillation in the ratio is due to the inaccurate field measurement close to the dipole source. Theoretically, we do not expect to see any difference at these offsets as what the other synthetic examples show in this paper. Note that on the negative offset side $(x<0)$, no difference in the measured field is observed because there is no reservoir on that side. This consistency of the measured field is an indication that the background geology is relatively uniform in this area.

Next, we construct a $4 \mathrm{~km}$ synthetic aperture source with no field steering. The fields with and without the reservoir are shown by the dashed and solid curves in Figure 15b, respectively. The

a)

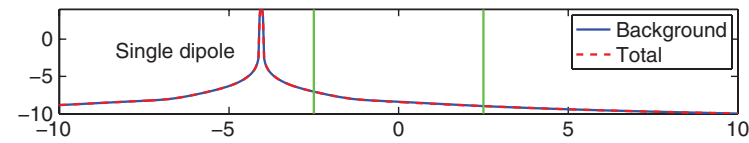

b)

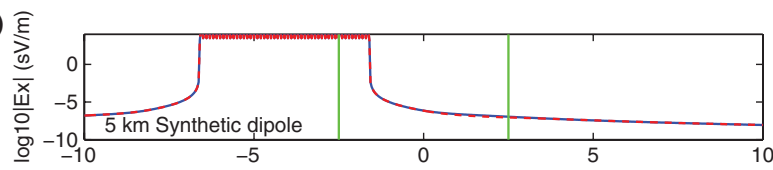

c)
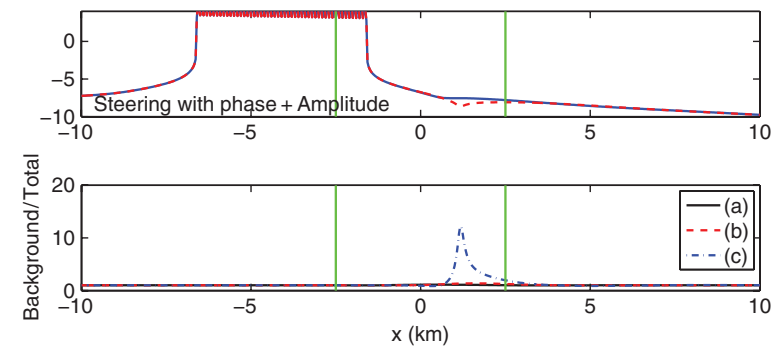

Figure 14. Panels (a-c) show the inline electrical field with the reservoir (dashed lines) and without the reservoir (solid lines) for three different sources; a single dipole source (a); a $5 \mathrm{~km}$ synthetic source (b); a $5 \mathrm{~km}$ synthetic source obtained from field steering toward the reservoir by the phase shift and the amplitude compensation (c). Panel (d) shows the ratio between the fields with and without the reservoir. The three curves in (d) represent the ratios from each of the panels above. In this system, both the dipole source and receivers are at the sea surface. corresponding ratio is the dashed curve in panel (d). Because the longer dipole source has a better signal to noise ratio, the $E_{x}$ field and the ratio are smoother than those from an individual source. The overall difference between the responses, however, does not change.

We next steer the fields toward the reservoir using a phase shift $\left(c_{1}=0.8\right)$ and amplitude weighting $\left(c_{2}=0.6\right)$. Figure $15 \mathrm{c}$ shows that the difference in the field between the models with and without the reservoir has significantly increased after we apply the field steering. The corresponding ratio is shown by the dashed-dotted line in panel (d). The imprint of the reservoir is much more pronounced in panel (c) than those in panels (a) and (b). Note that the response at negative offsets does not show any difference in the field before and after the field steering. This is because there is no reservoir for negative offsets. In this example, the choice of $c_{1}$ and $c_{2}$ is slightly different from the synthetic examples we showed above. The character of the anomaly is not the same as the synthetic examples above. There are multiple reasons that cause these differences, including changes in water depth, target location, target size and shape, background resistivity, possible resistive anisotropy, and the presence of noise. The sharp peak in the ratio anomaly (e.g., Figure 4e) is caused by the destructive dip in the background field (Figure 4e). This sharp dip in the background field may be filled by the noise, which corresponds to a smoother variation and lower value in the ratio anomaly. In general, different earth models give anomalies with different characteristics.

\section{SYNTHETIC VERTICAL SOURCE}

As we state in the shallow target synthetic example, the $z$-component of the $E$ field is sensitive to the changes in the conductivity in the vertical direction (Edwards, 2005). Therefore, a vertical oriented dipole source is most efficient to detect the horizontal reservoirs because the electrical field lines from a vertical source

a)

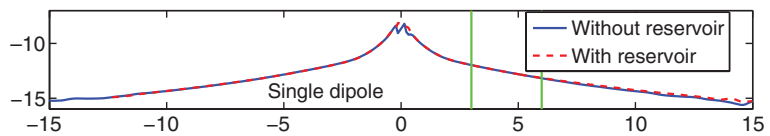

b)

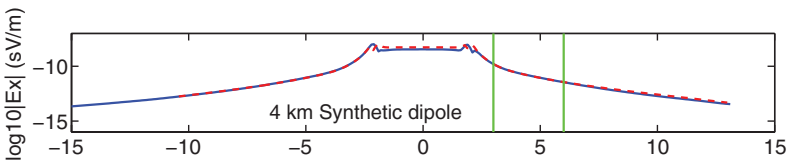

c)

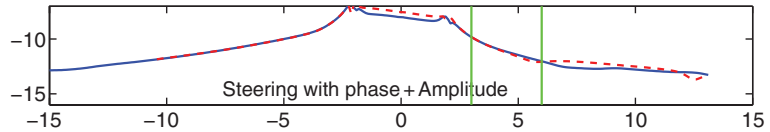

d)

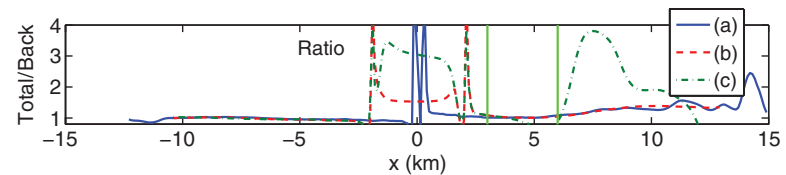

Figure 15. Panels (a-c) show the inline electrical field with the reservoir (red dashed lines) and without the reservoir (black solid lines) for three different sources; a single dipole source (a); a $4 \mathrm{~km}$ synthetic source (b); a $4 \mathrm{~km}$ synthetic source obtained from field steering toward the reservoir by the phase shift and the amplitude compensation (c). Panel (d) shows the ratio between the fields with and without the reservoir. The three curves in (d) represent the ratios from each of the panels above. 
have a large $z$-component (Mogilatov and Balashov, 1996; Holten et al., 2009). In practice there are many reasons that make it difficult to use a vertical dipole as a source. First, to maintain the vertical position of the $100 \mathrm{~m}$ long dipole is technically challenging. Second, the survey time, as well as the cost, can dramatically increase because of the difficulty in moving a vertical source.

A technique to generate a pseudovertical source with radiation pattern similar to that from a vertical source has been presented earlier (Balashov et al., 2001; Srnka and Carazzone, 2003; Helwig et al., 2010). The basic idea is that the similar radiation pattern as the vertical source can be constructed by adding orthogonally distributed horizontal dipole pairs, as illustrated in Figure 16. In this section, we show that the physical long dipoles used in these systems can be constructed synthetically by adding the response from a moving small dipole source. The synthetically constructed source provides the freedom to adjust the interference pattern of these orthogonal dipoles by field steering. In this paper, we refer the synthetic dipole array as the "synthetic vertical source."

In the following example, we apply the synthetic vertical dipole to a land acquisition system. A $100 \mathrm{~m}$ thick reservoir (resistivity of $100 \Omega \mathrm{m}$ ) with a horizontal extension of $4 \mathrm{~km}$ in $x$ - and $y$-directions, is located $1 \mathrm{~km}$ below the surface. The subsurface is a half space with a resistivity of $2 \Omega \mathrm{m}$. Figure 17 is a map view of the reservoir position (block in the center) and the four-dipoles system. Each dipole is $2 \mathrm{~km}$ long and centers of the two opposite dipoles are $10 \mathrm{~km}$ apart. We construct these $2 \mathrm{~km}$ long dipoles synthetically by adding the response from a moving $200 \mathrm{~m}$ long dipole.

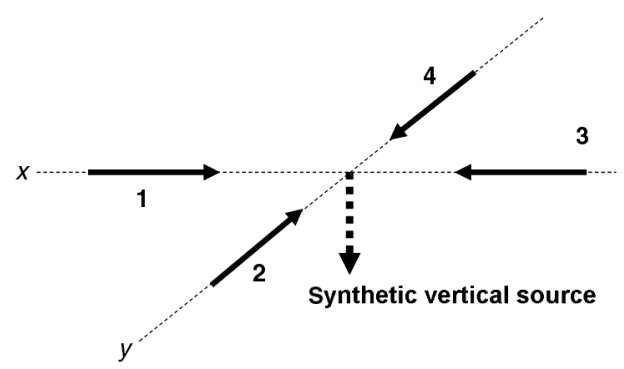

Figure 16. "Synthetic vertical source" (dashed arrow) construction by using two pairs of orthogonal dipoles (solid arrows).

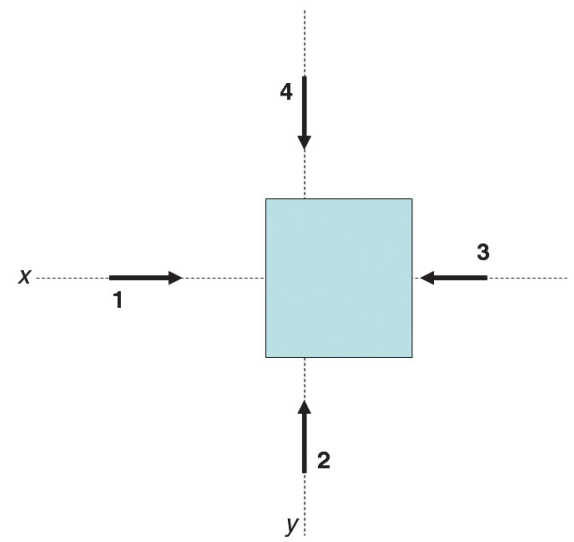

Figure 17. Map view of the reservoir (block in the center) and four dipoles.
The upper left and right panel of Figure 18, respectively show the cross section of the background $E_{x}$ and $E_{z}$ field in the $(x, z)$-plane from the four-dipole system. The $E_{x}$ field vanishes at $x=0$ because the cancellation of the fields from the four dipoles. The $E_{z}$ component excites the secondary field from the horizontal resistive target. The middle left and right panels show the secondary $E_{x}$ and $E_{z}$ field from the target, respectively. Because the background $E_{x}$ field is weak in the middle of the dipole array, the secondary field shows a strong anomaly in this area. The bottom left panel shows the measured $E_{x}$ field at the surface. The solid line is the background field and the dashed line is the total field (in the presence of the reservoir). The ratio of the above fields (with and without the reservoir) at the surface level is present in the bottom right panel. A strong anomaly is present in the center of the array. This anomaly is due to the destructive interference of the background $E_{x}$ field (dark color in the first panel of Figure 18) and constructive interference of the $E_{z}$ field (top right panel of Figure 18).

Next, we show that the cancellation of the $E_{x}$ field can be enhanced by field steering. Such steering can not be achieved if a physical $2 \mathrm{~km}$ long dipole is used. In our example, each $2 \mathrm{~km}$ long dipole is constructed by ten spatially distributed small dipoles that are $200 \mathrm{~m}$ long. These ten spatially distributed dipoles can be obtained by moving one dipole to ten different locations. The field from each $2 \mathrm{~km}$ long dipole can be steered using the method we proposed in the previous sections. Here, we steer the field of each dipole toward the center of the dipole array (toward the reservoir). Dipoles one and three are steered by the same steering parameter $c_{1}$ (one to the right and one to the left), which may be different from the steering parameter for dipoles teo and four. No amplitude weighting parameter $c_{2}$ is used in this example. Figure 19 shows the field in the same pattern as Figure 18 in the case where dipoles one and three are steered with $c_{1}=0.5$ and dipoles two and four are steered with $c_{1}=1$. Compared with the case without steering (Figure 18), the top left panel of Figure 19 shows that the
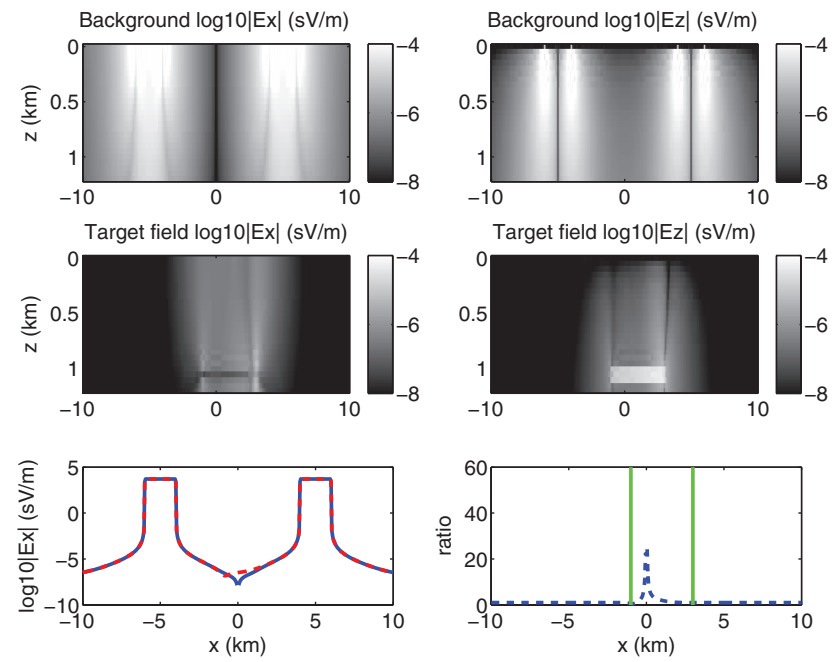

Figure 18. Top left: the cross section of the background $E_{x}$ field. Top right: the cross section of the background $E_{z}$ field. Middle left: the cross section of the target $E_{x}$ field. Middle right: the cross section of the target $E_{z}$ field. Bottom left: the background (solid) and total $E_{x}$ field (dashed) at the surface level along $x$-axis. Bottom right panel: the ratio of the total and the background $E_{z}$ field at the surface level. 

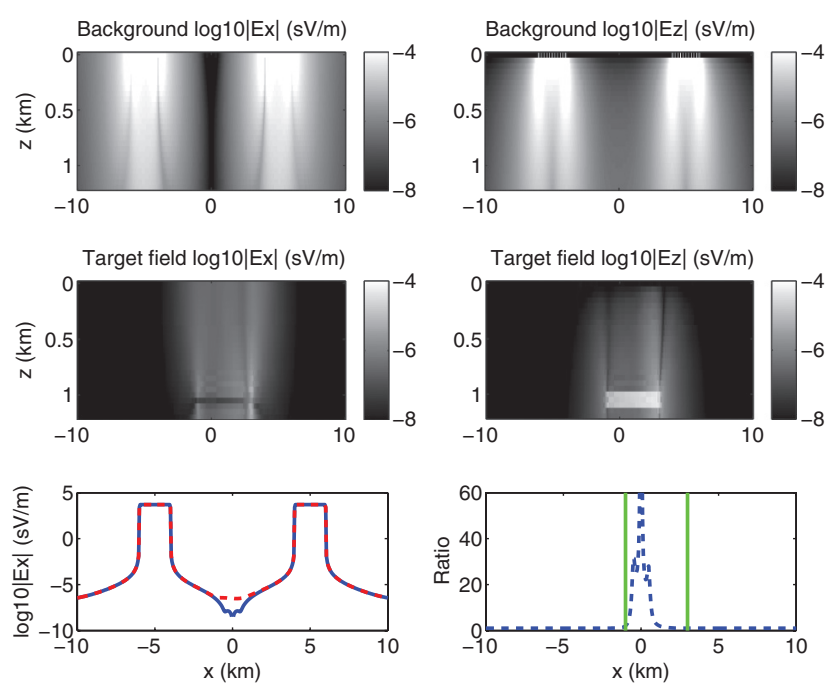

Figure 19. Top left: the cross section of the background $E_{x}$ field. Top right: the cross section of the background $E_{z}$ field. Middle left: the cross section of the target $E_{x}$ field. Middle right: the cross section of the target $E_{z}$ field. Bottom left: the background (solid) and total $E_{x}$ field (dashed) at the surface level along $x$-axis. Bottom right panel: the ratio of the total and the background $E_{z}$ field at the surface level.

background $E_{x}$ field in the center part becomes much smaller, while the middle left panel shows the strength of the secondary $E_{x}$ field from the target remains roughly unchanged. Therefore, the difference of the measured field at the surface level for the models with and without the reservoir, shown in the bottom left panel, is more pronounced because of the steering. The large anomaly in the bottom right panel of Figure 19 shows a much stronger imprint of the reservoir.

In the synthetic modeling study, we find that there are multiple pairs of steering parameters (beside $c_{1}=0.5$ for dipoles one and three and $c_{1}=1$ for dipoles two and four) which give a large anomaly in the measured field. The key is to adjust the fields from each dipole in such a way that the horizontal fields from each dipole interferes destructively. Beside the field steering, other parameters affect the strength from each source. These include the size of each dipole, the subsurface structure, and the location of the target. Further research is needed to optimize the construction of a synthetic vertical source. We only use two pairs of dipole sources in the above example, but it is possible to use more pairs of the dipoles to better force the field lines go vertically, such as the eight-dipole system used by Helwig et al. (2010). Because we use a synthetic aperture, more pairs of dipoles can be easily constructed by moving the small dipole source to new locations.

We have shown an application of the synthetic vertical source for a land system. In the marine case, constructing a synthetic vertical source can be implemented by adding a crossline sailing direction over the target area. Dipoles with opposite orientations can be obtained by reversing the sign of measured fields. The position and the size of the long synthetic dipole source can also be adjusted by changing the number and positions of the small dipoles.

\section{CONCLUSION}

We have shown that the detectability of hydrocarbon can be significantly increased by applying the synthetic aperture concept to CSEM data. This means that more challenging reservoirs can be detected. We also showed that the drawback (reduced sensitivity) of a streamer system can be reduce by applying field steering. The diffusive nature of electromagnetic fields in the conductive subsurface makes those fields decay rapidly in space, and as a consequence the target field, which carries useful information of the reservoir, is normally buried in the background field. Interpreting the presence of a hydrocarbon reservoir can thus be challenging. This difficulty restricts the applicability of CSEM to reservoirs which have large size, are shallow in depth, and have significant resistivity contrast with the surrounding subsurface. The best way to increase the detectability of reservoirs using CSEM is to simultaneously increase the target field and reduce the background field.

Using the synthetic aperture technique, large synthetic sources can be constructed by adding small sources in such a way that the total field from this synthetic source excites a large target field and a small background field for particular offsets. By doing so, significant reservoir anomalies can be found in measured fields. These anomalies not only help interpret the presence of the reservoir, but also increase the accuracy and speed of a CSEM inversion. We only show a few applications for field steering, but this does not suggest that this the only, or the optimal, way of applying the synthetic aperture technique.

Beside the application to horizontal electrical dipoles shown in this work, the same concept has the potential to be extended to other type of sources, such as the electrical loops used in airborne systems. We have steered the field by applying a linear phase shift in the source array. Focusing the field by applying quadratic phase shifts could, in principle, increase the secondary field generated by the target. A 2D source array would not only allow for steering the field in the inline direction, but also for steering in the crossline direction. We have demonstrated construction of synthetic aperture sources, but one can also construct synthetic aperture receivers. There are many opportunities to further enhance the use of a synthetic aperture source in CSEM, and one may learn these processes from synthetic aperture techniques used in the radar and sonar communities.

\section{ACKNOWLEDGMENTS}

The authors are grateful for the financial support through a Shell Gamechanger Project. The electromagnetic research team in Shell provided technical support in the research and we thank them for the permission for using the real data. The authors would like to express special thanks to the reviewers of this paper for their constructive suggestions and comments.

\section{REFERENCES}

Aguttes, J. P., J. Sombrin, E. Conde, M. Chaubet, I. Sebbag, and P. Bousquet, 2000, Radar "sail" satellite concept and design: Acta Astronautica, 46, no. 9, 565-576, doi: 10.1016/S0094-5765(00)00018-7.

Anderson, C., and J. Mattson, 2010, An integrated approach to marine electromagnetic surveying using a towed streamer and source: First Break, 28, no. 5, 71-75.

Balashov, B. P., V. S. Mogilatov, G. V. Sachenko, and A. K. Zakharkin, 2001, Method of prospecting for geological formations and apparatus for implementing the method: U. S. Patent 6,320,386 .

Barber, B. C., 1985, Theory of digital imaging from orbital syntheticaperture radar: International Journal of Remote Sensing, 6, no. 7, 1009-1057, doi: 10.1080/01431168508948262.

Bellettini, A., and M. Pinto, 2002, Theoretical accuracy of synthetic aperture sonar micronavigation using a displaced phase-center antenna: IEEE 
Journal of Oceanic Engineering, 27, no. 4, 780-789, doi: 10.1109/JOE .2002 .805096 .

Berson, M., A. Roncina, and L. Pourcelota, 1981, Compound scanning with an electrically steered beam: Ultrasonic Imaging, 3, no. 3, 303-308, doi: 10.1016/0161-7346(81)90162-0.

Boas, D. A., M. A. O'Leary, B. Chance, and A. G. Yodh, 1993, Scattering and wavelength transduction of diffuse photon density waves: Physical Review E (Statistical Physics, Plasmas, Fluids, and Related Interdisciplinary Topics), 47, no. 5, R2999-R3002, doi: 10.1103/PhysRevE .47.R2999.

Boas, D. A., M. A. O'Leary, B. Chance, and A. G. Yodh, 1994, Scattering of diffuse photon density waves by spherical inhomogeneities within turbid media: Analytic solution and applications: Proceedings of the National Academy of Sciences of the United States of America, 91, no. 11, 4887-4791, doi: 10.1073/pnas.91.11.4887.

Constable, S., and L. J. Srnka, 2007, An introduction to marine controlledsource electromagnetic methods for hydrocarbon exploration: Geophysics, 72, no. 2, WA3-WA12, doi: 10.1190/1.2432483.

Cutrona, L., 1975, Comparison of sonar system performance achievable using synthetic aperture techniques with the performance achievable by more conventional means: Journal of the Acoustical Society of America, 58, no. 2, 336-348, doi: 10.1121/1.380678.

Edwards, N., 2005, Marine controlled source electromagnetics: Principles, methodologies, future commercial applications: Surveys in Geophysics, 26, 675-700

Ellingsrud, S., T. Eidesmo, S. Johansen, M. C. Sinha, L. M. MacGregor, and S. Constable, 2002, Remote sensing of hydrocarbon layers by seabed logging (SBL): Results from a cruise offshore Angola: The Leading Edge, 21, 972-982, doi: 10.1190/1.1518433.

Fan, Y., R. Snieder, and J. Singer, 2009, 3D controlled source electromagnetic (CSEM) interferometry by multi-dimensional deconvolution: 79th Annual international Meeting, SEG, Expanded Abstracts, 779-784.

Fan, Y., R. Snieder, E. Slob, J. Hunziker, and J. Singer, 2011, Steering and focusing diffusive fields using synthetic aperture: Europhysics Letters, 95, no. 3, 34006, doi: 10.1209/0295-5075/95/34006.

Fan, Y., R. Snieder, E. Slob, J. Hunziker, J. Singer, J. Sheiman, and M. Rosenquist, 2010, Synthetic aperture controlled source electromagnetics: Geophysical Research Letters, 37, no. L13305, L13305, doi 10.1029/2010GL043981.

Filatov, V. V., 1984, Construction of focusing transformations of transient electromagnet fields: Geologiya i Geozika, 25, 85-89.

Helwig, S. L., V. S. Mogilatov, and B. P. Balashov, 2010, Enhanced sensitivity in land em by using an unconventional source: EGM 2010 International Workshop.

Holten, T., E. G. F. y, B. Singer, E. M. Blixt, A. Hanssen, and K. J. Måløy, 2009 , Vertical source, vertical receiver, electromagnetic technique for offshore hydrocarbon exploration: First Break, 27, no. May, 89-93.

Hunziker, J., Y. Fan, E. Slob, K. Wapenaar, and R. Snieder, 2010, Solving spatial sampling problems in 2D-CSEM interferometry using elongated sources: 72nd Annual International Conference and Exhibition, EAGE, Expanded Abstracts, 278-285.

Hunziker, J., E. Slob, and K. Wapenaar, 2009, Controlled source electromagnetic interferometry by multidimensional deconvolution: Spatial sampling aspects in sea bed logging: Annual International Conference and Exhibition, EAGE, Extended Abstracts, 278-285.

Isaev, G. A., and V. V. Filatov, 1981, Physicomathematical principles of visualization of nonstationary electromagnetic fields: Geologiya i Geozika, 22, 89-95.

Knuttel, A., J. Schmitt, R. Barnes, and J. Knutson, 1993, Acoustooptic scanning and interfering photon density waves for precise localization of an absorbing (or fluorescent) body in a turbid medium: Review of Scientific Instruments, 64, no. 3, 638-644, doi: 10.1063/ 1.1144190 .
Korobov, A. I., M. Y. Izosimova, and S. A. Toschov, 2010, Development of ultrasound focusing discrete array for air-coupled ultrasound generation Physics Procedia, 3, no. 1, 201-207, doi: 10.1016/j.phpro.2010.01.028.

Kunetz, G., 1972, Processing and interpretation of magnetotelluric sounding: Geophysics, 37, 1005-1021, doi: 10.1190/1.1440310.

Lee, K. H., G. Liu, and H. F. Morrison, 1989, A new approach to modeling the electromagnetic response of conductive media: Geophysics, 54, 1180-1192, doi: 10.1190/1.1442753.

Lu, J.-Y., H. Zou, and J. F. Greenleaf, 1994, Biomedical ultrasound beam forming: Ultrasound in Medicine \& Biology, 20, no. 5, 403-428, doi: $10.1016 / 0301-5629(94) 90097-3$

MacGregor, L., and M. Sinha, 2000, Use of marine controlled-source electromagnetic sounding for sub-basalt exploration: Geophysical Prospecting, 48, 1091-1106, doi: 10.1046/j.1365-2478.2000.00227.x.

McKay, A. J., C. Clarke, J. Linfoot, and J. Mattsson, 2011, Interpretative quality control of towed EM data - Examples from the North Sea: 73rd Annual International Conference and Exhibition, EAGE, Extended Abstracts, I006.

Mogilatov, V., and B. Balashov, 1996, A new method of geoelectrical prospecting by vertical electric current soundings: Journal of Applied Geophysics, 36, no. 1, 31-41, doi: 10.1016/S0926-9851(96)00030-4.

O'Leary, M. A., D. A. Boas, B. Chance, and A. G. Yodh, 1992, Refraction of diffuse photon density waves: Physical Review Letters, 69, no. 18 , 2658-2661, doi: 10.1103/PhysRevLett.69.2658.

Ralston, T. S., D. L. Marks, C. P. Scott, and S. A. Boppart, 2007, Interferometric synthetic aperture microscopy: Nature Physics, 3, no. 2, 129-134 doi: $10.1038 /$ nphys514.

Riyait, V., M. Lawlor, A. Adams, O. Hinton, and B. Sharif, 1995, Real-time synthetic aperture sonar imaging using a parallel architecture: IEEE Transactions on Image Processing, 4, no. 7, 1010-1019, doi: 10.1109/ 83.392341

Schmitt, J. M., A. Knuttel, and R. F. Bonner, 1993, Measurement of optical properties of biological tissues by low-coherence reflectometry: Applied Optics, 32, no. 30, 6032-6042, doi: 10.1364/AO.32.006032.

Schmitt, J. M., A. Knuttel, and J. R. Knutson, 1992, Interference of diffusive light waves: Journal of the Optical Society of America, 9, no. 10 1832-1843, doi: 10.1364/JOSAA.9.001832.

Srnka, L. J., and J. J. Carazzone, 2003, Method of imaging subsurface formations using a virtual source array: U.S. Patent No: US 2003/0050759 A1.

Wang, C., and A. Mandelis, 1999, Purely thermal-wave photopyroelectric interferometry: Journal of Applied Physics, 85, no. 12, 8366-8377, doi: 10.1063/1.370684.

Wang, W.-Q., J. Cai, and Q. Peng, 2009, Conceptual design of near-space synthetic aperture radar for high-resolution and wide-swath imagingstar: Aerospace Science and Technology, 13, no. 6, 340-347, doi: 10.1016.j.ast 2009.06.006.

Wapenaar, K., E. Slob, and R. Snieder, 2008, Seismic and electromagnetic controlled-source interferometry in dissipative media: Geophysical Prospecting, 56, 419-434, doi: 10.1111/j.1365-2478.2007.00686.x.

Yodh, A., and B. Chance, 1995, Spectroscopy and imaging with diffusing light: Physics Today, 48, no. 3, 34-40, doi: 10.1063/1.881445.

Zhdanov, M. S., and P. Traynin, 1997, Migration versus inversion in electromagnetic imaging technique: Journal of Geomagnetism and Geoelectricity, 49, no. 11-12, 1415-1437, doi: 10.5636/jgg.49.1415.

Zhdanov, M. S., P. Traynin, and J. R. Booker, 1996, Underground imaging by frequency domain electromagnetic migration: Geophysics, 61, 666-682, doi: 10.1190/1.1443995.

Zhdanov, M. S., P. Traynin, and O. Portniaguine, 1995, Resistivity imaging by time domain electromagnetic migration (TDEMM): Exploration Geophysics (Collingwood, Australia), 25, 186-194.

Zhou, X., N. Chang, and S. Li, 2009, Applications of SAR interferometry in earth and environmental science research: Sensors, 9, no. 3, 1876-1912, doi: $10.3390 / \mathrm{s} 90301876$. 